University of Warwick institutional repository

This paper is made available online in accordance with

publisher policies. Please scroll down to view the document

itself. Please refer to the repository record for this item and our

policy information available from the repository home page for further information.

To see the final version of this paper please visit the publisher's website. Access to the published version may require a subscription.

\begin{tabular}{|c|c|}
\hline Authors: & R E Klaber, C F Macdougall \\
\hline Title: & $\begin{array}{l}\text { Maximising learning opportunities in } \\
\text { handover }\end{array}$ \\
\hline $\begin{array}{l}\text { Year of } \\
\text { publication: }\end{array}$ & 2009 \\
\hline $\begin{array}{l}\text { Link to } \\
\text { published } \\
\text { version: }\end{array}$ & http://dx.doi.org/10.1136/adc.2008.142471 \\
\hline $\begin{array}{l}\text { Publisher } \\
\text { statement: }\end{array}$ & None \\
\hline
\end{tabular}




\title{
Maximising learning opportunities in handover
}

\author{
R E Klaber, ${ }^{1,2}$ C F Macdougall ${ }^{3}$
}

\begin{abstract}
${ }^{1}$ Department of Paediatrics, St Mary's Hospital, Imperial College Healthcare NHS Trust, London, UK; ${ }^{2}$ London Specialty School of Paediatrics and Child Health, London, UK; ${ }^{3}$ Warwick Medical School and University Hospital, Coventry, UK

Correspondence to:

Dr R E Klaber, Department of Paediatrics, St Mary's Hospital, Imperial College Healthcare NHS Trust, London, UK; bobklaber@ doctors.org.uk
\end{abstract}

Accepted 26 February 2009
Handing over responsibility for patients has always been part of medical practice. Definitions emphasise transfer of responsibility to ensure patient safety and the available literature tends to follow this line (see box 1). Handover is much more than this, however. It is a key event where teams meet, have the opportunity to communicate, support each other and learn. This paper considers different ways of maximising learning opportunities in handover, with particular emphasis on the strengths and challenges of the paediatric environment. Alongside review of the best available evidence, many of the ideas discussed were generated from working with a group of 65 experienced paediatricians with particular experience and interest in medical education as part of the Royal College of Paediatrics and Child Health Paediatric Educators Programme.

Formal handover has increased in importance and been embedded in practice with the transition from "on-calls" to "full-shift" rotas in an effort to comply with the European Working Time Directive $^{1}$ in the United Kingdom (UK). Departments responsible for acute patient care have had to incorporate two or three handover sessions into every day to ensure patient problems and management plans are appreciated by the incoming medical team.

Surveys have been conducted to look at handover practices $^{2-4}$ and to consider how changes, perceived to improve practice, can be implemented. It is widely acknowledged that the primary aim of handover is to ensure effective continuity of care for patients, and a number of guidelines and frameworks have been published to promote this. ${ }^{5-10}$ The focus is mainly on clinical risk management and governance, with emphasis on good communication and prioritisation of clinical issues. With evidence suggesting that the quality of handover is often poor, ${ }^{2-4}$ the development of an understanding of how mistakes in handover can lead to serious failures can jump-start initiatives to improve patient safety. ${ }^{11}$ Most of these initiatives have been developed with help from outside medicine using, for example, expertise from space, nuclear, aviation and motor-racing industries. ${ }^{11} 12$

\section{Box 1 Definition of "handover"}

Handover is "the transfer of professional responsibility and accountability for some or all aspects of care for a patient, or group of patients, to another person or professional group on a temporary or permanent basis" (The National Patient Safety Agency²).
Documentation and reporting formats for handover have also been examined. Van Eaton et a ${ }^{13}$ demonstrated that an automated computerised patient data system, designed to support handover, enhanced the quality and continuity of patient care. The World Health Organization is promoting a standardised approach to handover communications using the SBAR (Situation, Background, Assessment and Recommendation) questioning technique, which specifies the type of information that needs to be communicated at handover. ${ }^{14}$ There are also examples of locally-designed documentation-based handover tools which have been developed to support patient safety..$^{15}$ There is likely to be further expansion in the use of these types of tool and as electronic patient records become further established in the use of electronic handover aids.

The potential educational value of handover, however, is barely mentioned in current literature. Two recent studies within emergency departments $^{16}{ }^{17}$ recommend incorporating handover practices into the departmental education programme although they provide little detail of how this might be achieved.

\section{HANDOVER IN THE PAEDIATRIC ENVIRONMENT}

Within the working environments of different paediatricians the structure, frequency and duration of handover varies hugely. It may take the form of informal office-based discussions, informal ward rounds, multidisciplinary meetings or full departmental meetings and formal "lecture type" presentations. In addition to differences in working practices between emergency room, intensive care, community, general and specialist paediatricians, many units have developed a mixture of handover practices appropriate to different times of day or days of the week. These complexities have important implications when considering how to plan handover, set appropriate standards and ensure that colleagues are appropriately trained to deliver them.

Of particular relevance to paediatricians, inpatient paediatrics involves short stays and rapid turnover where most of the patients are new to the incoming team. Anderson ${ }^{4}$ compared clinical handover practices in general paediatric units across three postgraduate deaneries in the UK with two of the published guidelines. ${ }^{57}$ There was a significant unmet need for training in the practice of handover with only three out of 24 units providing departmental teaching on handover. In terms of using handover as an opportunity to learn, 14 units 
reported that handover time was used for additional educational activities varying from planned topics to identifying key experiential learning points, or as labelled by one unit "clinical pearls".

\section{UNDERPINNING EDUCATIONAL CONCEPTS \\ Context}

Medical education in the UK has been in a state of flux for some years. The national agenda has been a shift from care provided by those in training to provision by the trained ${ }^{18}$ and the adoption of the European Working Time Directive ${ }^{1}$ has changed working practices in acute services beyond recognition. There are now more trainees needing to learn more quickly:

the knowledge, skills and techniques which trainees now have to acquire are greater than in the past and have to be acquired during a much shorter training. ${ }^{19}$

The reaction to the pressures of providing "concentrated learning" has often been to pull trainees from service and "teach them" despite the evidence that professionals learn best by being part of the activity they are learning:

adults are likely to learn more effectively when the learning tasks are seen to be relevant, meaningful, interesting and useful. ${ }^{20}$

While it is clearly possible to design classroom teaching that addresses these principles, learning as part of working is imbedded in what the learner needs and wants. Immersing someone in work is not enough for them to learn, however. Good learning is integrated with work, not replaced by it:

on the job training needs to be: planned rather than opportunistic, fusional rather than intrusive, cyclical rather than fragmented, an investment rather than a duty. ${ }^{19}$

The challenge is therefore to provide teaching that is planned, fusional and cyclical in a way that is interesting, useful and meaningful if we are to successfully invest in our trainees. Handover provides a feasible if challenging area within which to ensure an educational opportunity in clinical practice is not missed.

\section{Planned}

Education does not happen by accident, although the experience around which good learning happens can be unpredictable. Handover is one element of the clinical day that is predictable and is therefore more amenable to planning for learning than, for example, practical medical emergencies.

\section{Fusional}

Learning should be seen as integral to care, not a separate entity alongside it. Handover should involve summarising cases, reporting clinical assessments, investigations, completed and planned actions and formulating plans for the shift ahead. There may also be opportunities to explore theoretical extrapolations (what if I had managed the patient this way instead of that?). Such a cycle of action, reflection, extrapolation and planning mirrors learning models, such as Kolb's learning cycle. $^{21}$

\section{Cyclical}

Handover is a regular feature in acute units. This gives real potential for cyclical and developmental learning. For example, a challenging patient may be presented in the morning, with discussion resulting in a particular management plan. At afternoon handover, the team can catch up on the case and learn from the progression of the situation. The challenge is to maximise these opportunities and develop strategies for when key learners (such as those who worked overnight) are not at the next handover.

\section{Interesting}

For trainees to value learning at handover, they need to be enthusiastic about going beyond the necessary basics. We are fortunate to generally work with motivated and experienced learners. Getting the balance between riding the wave of this enthusiasm and frustrating a group of tired doctors is important.

\section{Useful}

Trainees need to feel the learning in handover has benefited them and their learning needs. This should be straightforward as the focus is around a group of patients that they have already been managing or are about to manage and discussions will be of direct relevance to their work (see boxes 2 and 3). The balance for the teacher is between positively picking up on the "clinical pearls"4 and expanding the discussion to widen learning, or more negatively, using handover as a forum for their own hobby horses or narrow clinical preoccupations.

\section{Meaningful}

In making learning meaningful there needs to be alignment between the aims of what the individual or department is trying to teach, what the trainee feels they need, what actually happens and how it is assessed - so-called constructive alignment. ${ }^{26}$

For example, if a department decides to engender reflection of difficult overnight emergency cases but trainees are more anxious about the complex chronic patients, frustration will ensue. If trainees find that either their discussions are curtailed or that raising such issues is seen as demonstrating a lack of clinical confidence, they will become disillusioned. If they put thought and effort into handover that is then rushed or cancelled, they will become resentful.

\section{Return on investment}

The danger in all this is that clinicians will see handover as just another in a long list of tasks in an 


\section{Box 2 Giving feedback}

Monday morning handover. The team who have been working three night shifts over the weekend are handing over to a group of 10 consultant and trainee colleagues. It is clear to the attending consultant that an error has been made overnight by the night team. This error has not led to any significant clinical implications in this particular case, but in another situation it may have done. When is the best time to highlight this error, and who should be present when feedback is given? One of the main challenges of incorporating learning into handover is giving feedback, and indeed, two surveys of the senior house officer grade have highlighted shortcomings in feedback following outof-hours work. ${ }^{22} 23$

The learning potential versus patient safety needs balance and may sometimes conflict, for example, the need to ensure a team understands there has been an error (so that they don't repeat it and the day team can help correct it) versus the educational context of using error to teach.

The timing, content and approach of feedback have to be carefully handled. Handover often involves the more junior and often most tired members of the team discussing their patient assessment and management skills with the more senior and often better rested. If trainees are to benefit from discussion and critique of their actions and the team is to learn from the successes and challenges of the last shift, then a constructive, appropriately challenging, but blame-free culture is required. Trainees must feel able to discuss issues openly expecting support, understanding and guidance of how to do better next time, rather than accusation or humiliation.

Feedback immediately after a shift has finished has advantages of being current and helping guide reflection. Difficulties include finding sufficient time when clinical matters (eg, a ward round) are pending and finding an appropriate environment for individual feedback, particularly when this may be sensitive for the individual yet a learning opportunity for the whole team. In some cases feedback will provide helpful closure and in others stimulate anxiety and upset at a time when trainees should be resting prior to their next shift. Alternatively, feedback can be given at a later date, but in reality these opportunities are often not taken and teamlearning opportunities can be missed.

otherwise busy day. Yet helping trainees to learn how to properly treat, manage and hand over care has real potential for reducing workloads by ensuring confidence in delegation, providing a mechanism for oversight of action and potentially improving patient safety and care.

PUTTING EDUCATION INTO HANDOVER PRACTICE Handover has the potential to be the champion of good work based learning. It is a regular event

\section{Box 3 Assessment}

Concerns have been expressed about the abilities of a specialist registrar. Reported findings at handover seem to be at odds with what is found on the ward round and it is unclear whether this is a weakness in clinical ability or in handing over that information. How could handover skills be assessed? Could this be an area covered by a work-based assessment tool?

The modern UK zeitgeist is that the key to improvement (whether it be in primary school learning, health care delivery or provision of public utilities) is measurement, often in comparison to targets. Within medical education this has seen a huge rise in techniques aimed at assessing competence and performance in the workplace. ${ }^{24} 25$ However, we do not currently formally assess abilities to handover. The oft quoted mantra that "assessment drives learning" suggests that if we don't assess handover, it will not be valued, although, as noted, there are other ways of a department emphasising that handover skills matter.

Certainly, a handover assessment tool could be devised to join the clinical assessment toolkit and this could be used formatively to help develop trainee skills or summatively as part of college assessments. This could either focus on the clinical cases (based on case based discussion models) or on the management and leadership of the session. Development would however require agreement on one or more desirable models of handover and risks adding to the overall assessment burden of the clinical departments.

which involves review of clinical care in the preceding time period, synthesis of clinical cases and planning of both clinical (treatment and investigation) and practical (who will do what) issues. Trainees also need to be taught the operational and communication skills of handing over: prioritising of information, summarising, presenting and questioning skills.

This section gives some practical skills, tips and tools for taking on the challenges of handover and putting these concepts into practice. Improvements to practice will depend on what is already in place. Those starting from scratch may want to work initially with the key steps in box 4 .

\section{Planning}

Good handover doesn't just happen. Instigating and maintaining good practice needs leadership, planning, ownership and direction from lead clinicians. This needs preparation and reflection, ongoing review of the process and appropriate change as required.

Critically, the team needs to understand the purpose of handover and each type (eg, morning versus evening) needs clear objectives. This clarity should result in clear ground rules (eg, time-keeping/ structure/roles/focus/supporting documentation). A 


\section{Box 4 Key elements of learning in handover}

1. Departments need to have a consistent approach that handover is important and valued.

2. A clear structure is needed to reflect the local purposes of different handovers.

3. Trainees come with a variety of experiences that could help or hinder local practice - capturing experiences from elsewhere can be a valuable source of new ideas.

4. Handover skills need to be learned and improved during training and beyond.

5. There are many learning opportunities in handover but utilising these takes effort and planning.

6. There needs to be careful thought about how to give feedback during or around handover.

7. If handover is to improve, this needs ongoing reflection, audit and perhaps assessment.
Time constraints in handover are the major perceived barrier. ${ }^{27}$ Strategies include allocating a defined time within handover for teaching and running specific "teaching handovers" at a set point in the week, although both threaten perceived integration with clinical work. There are also well-established examples of time-efficient teaching strategies such as the "one-minute preceptor", ${ }^{28}$ where answers to clinical questions are explored for evidence and generalisability, that can have a key role within handover.

Topics encountered in clinical discussions during handover can be used as a driver for self-directed learning at a later stage (sometimes named "educational prescriptions" ${ }^{29}$ ). Alternatively, a team approach to learning can be developed using an "education book" - questions and discussions from handover are noted down and subsequently reviewed, researched and presented by the team later in the week.

The key advantage to immediately addressing learning needs within a handover is true "on the job" embedded learning. The challenge is doing it in such a way that is perceived as valuable rather than simply slowing down an otherwise busy clinical event. "Deferral" approaches such as learning prescriptions provide a degree of contextualisation but risk becoming an exercise in window dressing if the identified needs are not followed up.

The success of these initiatives depends upon the planning and organisation discussed earlier. The direct involvement of trainees in the process is key, both in terms of encouraging them to think about how to organise and run handover, as well as debriefing them and gaining feedback on their experiences at the end of their placements.

themselves to the handing on of basic factu information, although how much of this is retained is contentious. Key areas to cover are objectives and ground rules, documentation, timekeeping and structure.

Those involved in leading handover need to model good practice, provide leadership and direction, value the handover and the contribution of others as well as maximising the learning opportunities available for those present.

As with other areas of clinical practice, handover skills need to progressively develop as trainees gain seniority. They need to be allowed to lead handovers independently, perhaps using peer or video observation and promotion of reflection. There may also be a role for a handover assessment tool.

\section{Teaching and learning in handover}

Handover provides a daily opportunity for casebased learning with the chance for trainees to discuss clinical issues with senior colleagues in an open forum. Clinical findings, investigations, differential diagnoses, management plans and prioritisation can be discussed and fixed ideas about clinical cases challenged to encourage broad and open thinking. The evidence base and an exploration of the process of clinical reasoning and decision making can also be covered.

\section{POTENTIAL FURTHER IMPROVEMENTS TO EDUCATION WITHIN HANDOVER Key steps to improving handover}

Handover is right at the interface between service provision and education and is an area where these two essential components of medicine have to overcome difficulties to successfully work in tandem. There are, however, many simple ideas to improve practice in terms of patient safety but also maximise learning opportunity. Handover is here to stay - we need to do it well and make the most of the opportunity it provides. We accept that many of the recommendations aspire to producing the "perfect" handover. For those beginning to consider their practice and unclear where to start, the key elements are listed in box 4.

\section{Learning and personal development opportunities}

An important process for developing the overall quality of handover is to audit key areas which examine whether expected standards of handover are being consistently met. Audit questions may ask whether the handover has been appropriately prepared (ie, with results available/accurate 
written sheets), whether findings on the ward round match the information given in handover or if the numbers of communication-related clinical incident reports have reduced. ${ }^{12-15}$ There could also be focus on the quality of learning during handover, examining what trainees are learning during handover and the quantity and quality of the questions generated within handover and answered elsewhere. Other less formal development mechanisms are to encourage an environment for regular reflection on the handover process. Broekhuis and Veldkamp ${ }^{30}$ developed such a reflexivity method to reflect on clinical handover in a large university medical centre in the Netherlands, which was positively evaluated by users.

\section{CONCLUSIONS}

Irrespective of whatever else they learn during handover, trainees need to learn how to handover. Good handover practice in itself is an opportunity for modelling and "learning by doing". To know how to implement this we need some understanding of our learners, their learning needs, how learning takes place and how it can be promoted. Many of these are key skills taught, to some degree, as part of primary medical qualifications, although there is little evidence to suggest that they are being taught with handover specifically in mind. ${ }^{9}$ This should be fostered from an early stage by defining and actioning a role for medical students in handover.

Handover is one of the best opportunities in the working week for consultants to see trainees performing clinical work. This provides a forum for teaching and learning, mentoring, role-modelling professionalism, assessment (informal or with a structured assessment tool) and giving feedback. Handover skills need to be defined, taught and assessed, with clear standards and expectations presented to each new cohort of trainees at the beginning of their jobs.

Competing interests: None.

\section{REFERENCES}

1. Department of Health. European working time directive. See http://www.dh.gov.uk/en/Managingyourorganisation/ Humanresourcesandtraining/Modernisingworkforceplanninghome/ Europeanworkingtimedirective/index.htm (accessed 2 June 2009)

2. Roughton VJ, Severs MP. The junior doctor handover: current practices and future expectations. J $R$ Coll Physicians Lond 1996:30:213-14.

3. Sabir N, Yentis SM, Holdcroft A. A national survey of obstetric anaesthetic handovers. Anaesthesia 2006;61:376-80.

4. Anderson M. General paediatric clinical handover: passing the baton or passing the buck? Arch Dis Child 2006;91(Suppl 1):A74-7.

5. BMA. Safe handover: safe patients. See http://www.bma.org.uk/ images/safehandover_tcm41-20983.pdf.
6. Australian Commission on Safety and Quality in Health Care. Clinical handover and patient safety - May 2005. See http://www.health.gov.au/internet/safety/publishing.nsf/Content/ clinical-handover (accessed 2 June 2009).

7. RCPCH. Good practice in handover. See http://www.rcpch.ac.uk/ Publications/Publications-list-by-date (accessed 2 June 2009).

8. Royal College of Physicians. Guidelines on effective patient handover for physicians. In: General professional training guide. London: Royal College of Physicians, 2005:25. See http://www. rcplondon.ac.uk/pubs/handbook/gpt/GPTguide.pdf laccessed 2 June 2009).

9. Beasley R. From medical student to junior doctor: the medical handover - a good habit to cultivate. Student BMJ 2006;14:188-9.

10. Harrison M, Eardley W, McCarron B. Time to hand over our old way of working? Hosp Med 2005;66:399-400.

11. Patterson ES, Roth EM, Woods DD, et al. Handoff strategies in settings with high consequences for failure: lessons for health care operations. Int J Qual Health Care 2004;16:125-32.

12. Catchpole KR, de Leval MR, McEwan A, et al. Patient handover from surgery to intensive care: using Formula 1 pit-stop and aviation models to improve safety and quality. Paediatr Anaesth 2007:17:470-8.

13. Van Eaton EG, Horvath KD, Lober WB, et al. A randomized, controlled trial evaluating the impact of a computerized rounding and sign-out system on continuity of care and resident work hours. J Am Coll Surg 2005;200:538-45.

14. Institute for Healthcare Improvement. SBAR technique for communication: a situational briefing model. See http://www.ihi. org/lHI/Topics/PatientSafety/SafetyGeneral/Tools/ SBARTechniqueforCommunicationASituationalBriefingModel.htm (accessed 2 June 2009).

15. Brighton and Sussex University Hospitals NHS Trust. Risk Management annual report 2007. Available from www.bsuh.nhs. uk/EasySiteWeb/GatewayLink.aspx?alld=46888 (accessed 17 June 2009).

16. Ye K, McD Taylor D, Knott JC, et al. Handover in the emergency department: deficiencies and adverse effects. Emerg Med Australas 2007;19:433-41.

17. Jenkin A, Abelson-Mitchell N, Cooper S. Patient handover: time for a change? Accid Emerg Nurs 2007;15:141-7.

18. Department of Health. A health service of all the talents: developing the NHS workforce: consultation document on the review of workforce planning. London: Department of Health, 2000.

19. Hargreaves DH. On-the-job training for physicians: a practical guide. London: Royal Society of Medicine, 1997:xv, 151.

20. Daines J, Daines C, Graham B, eds. Adult learning, adult teaching. 3rd edn. Nottingham: Department of Adult Education, University of Nottingham, 1993.

21. Kolb DA. Experiential learning: experience as the source of learning and development. London: Prentice-Hall, 1984:xiii, 256.

22. Baldwin PJ, Newton RW, Buckley G, et al. Senior house officers in medicine: postal survey of training and work experience. BMJ 1997:314:740-3

23. Onyon C, Wall D, Goodyear H. Out-of-hours work: what is the training value for paediatric senior house officers? Br J Hosp Med 2007:68:100-1.

24. Davies H, Archer J, Heard S, et al. Assessment tools for foundation programmes - a practical guide. BMJ Career Focus 2005;330:195-6.

25. Crossley JGM, Howe A, Newble D, et al. Sheffield Assessment Instrument for Letters (SAIL): performance assessment using outpatient letters. Med Educ 2001;35:1115-24.

26. Bligh J, Prideaux D, Parsell G. PRISMS: new educational strategies for medical education. Med Educ 2001;35:520-1.

27. Irby DM, Wilkerson L. Teaching when time is limited. BMJ 2008;336:384-7.

28. Neher J0, Gordon KC, Meyer B, et al. A five-step "microskills" model of clinical teaching. J Am Board Fam Pract 1992:5:419-24.

29. Sackett DL, Haynes RB, Guyatt GH, et al. Keeping up to date - the educational prescription in clinical epidemiology. 2nd edn. Boston: Little Brown, 1991.

30. Broekhuis $\mathbf{M}$, Veldkamp C. The usefulness and feasibility of a reflexivity method to improve clinical handover. J Eval Clin Pract 2007:13:109-15. 